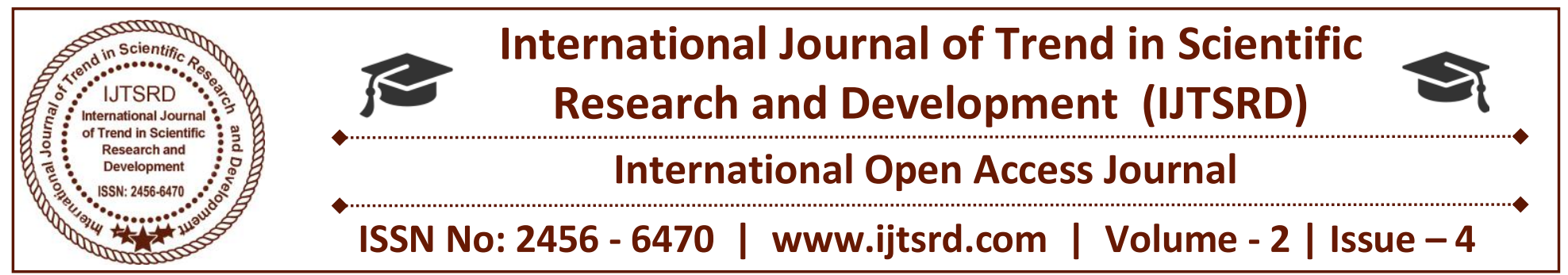

\title{
Blind Navigation: Technologies for Visually Impaired
}

\author{
Madhushree $\mathbf{M}^{1}$, Sushmitha $\mathbf{M}^{2}$, Manjunath $\mathbf{C} \mathbf{R}^{3}$, Soumya K $\mathbf{N}^{4}$ \\ ${ }_{1,2,4}$ Department of Information Science and Engineering, \\ ${ }^{3}$ Department of Computer Science and Engineering \\ 1,2,3,4 School of Engineering and Technology, Jain University, Bengaluru, Karnataka, India
}

Abstracts-- Blindness is a condition in which an individual loses the visual recognition. Mobility and self-reliability for the blind has dependably been an issue. A considerable measure has been made to propel advances that enhance blind and partial sighted individual's spatial observation. Many surveys of various technologies of navigation system for visually impaired with their usefulness, design and working according to the requirement of blind people is conducted. Current innovation gives applications to be proficiently appropriated and keep running on versatile and hand-held gadgets, even in cases wherever unique procedures are vital. Electronic travel aids navigation modules, text-to-speech applications, with added advancement in the technology are getting integrated into normal mobile devices. These technologies combined with continuous simple interfaces and methods of collaboration has opened a scope of most recent perspectives for the restoration and being easy to use of people with visual impairment. In general, it can be summarized to those technologies supporting the visually impaired with basic analysis and application development. This can provide better understanding of the technologies and their further analysis for future research.

Keywords-- Blindness; Technologies; Navigation System; Electronic travel aids;

\section{INTRODUCTION}

Blindness is the condition of being completely sightless in both eyes. Each visually impaired person can't analyze any event. The visual agnosia is usually used as a relative term to indicate disablement, or the low vision, which implies that even with eyeglasses, contact focal points, medication or then again surgery, an individual doesn't see well. Vision impedance will differ from fragile to extreme. India comes in the next range of blind folks at international forums due to its definition. India currently has around twelve million blind folks against thirty-nine million globally, that makes India home to simple fraction of the world's blind population. The estimated range of individuals who are visually impaired within the world is 300 to 400 million, among which 50 million are totally blind and few having low vision. Approximately $80 \%$ of visual impairment happens in individuals more than 50 years old. Regular reasons for visual impairment are complexities of diabetes, macular degeneration, horrendous wounds, contamination of the film or tissue layer, glaucoma, and powerlessness to get any glasses.

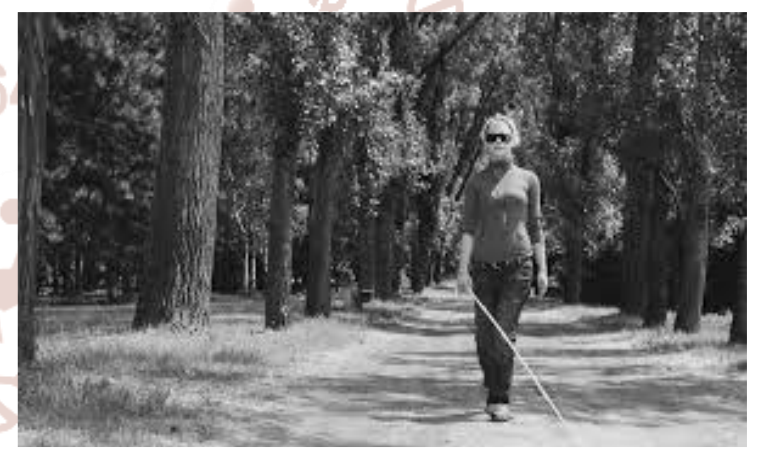

Figure 1: A blind person

Color blindness is that the inability to understand different variations in numerous colors, significantly green, red, then others will differentiate. It's most frequently familial and affect concerning $8 \%$ of males and $1 \%$ of females. Individuals who have partially blind, normally have customary vision and may perform well outwardly. This can't be extremely a genuine visual impairment. Night visual impairment could be an issue in observing things under 
diminished enlightenment. It is hereditary or nonheritable. According to World Health Organization, the huge number of individuals has visual modality difficulties but perform well underneath traditional lighting conditions. This cannot be a state of blindness.

Visual impairment or blindness can also be loss of vision when introduction of the eyes to certain measure of brightness. Visual impairment is ordinarily caused by swelling of cells of tissue layer of the eye. In most extreme instances of visual deficiency, the individual keeps on being prepared to see shapes and development. Ophthalmology is a claim to fame of solution those arrangements with determination and medicinal and surgical task of Page 2 infection. In this manner, ophthalmologists are the masters who have the learning and apparatuses to analyze visual deficiency and give treatment, if conceivable.

\section{Global Blindness}

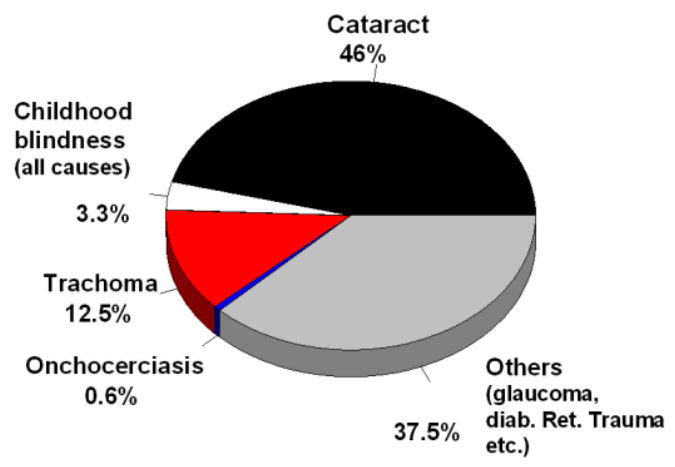

Figure 2: Global Blindness.

Some basic reasons for visual imperfection incorporate vitamin-A deficiency retinopathy of immaturity, sickness involving the tissue layer or second cranial nerve together with stroke, ocular disease, retinitis pigmentosa, primary or secondary malignancies of the eye, congenital abnormalities, hereditary diseases of the eye, and chemical poisoning from toxicant agents like methanol. There can be impermanent visual impairment which contrasts totally from changeless visual impairment. To look at the imperfection in eye, every one of the parts of the eye must be analyzed to check the deformity by an ophthalmologist.

Related side effects, such a distress inside the eyes, consciousness of the eyes, outside body sensation, and torment inside the eyes or release from the eyes is additionally blessing or missing, wagering on the basic purpose behind the sightlessness. A visually impaired man won't not have any noticeable indications of irregularities once sitting in a seat and resting. The most well-known side effects of visual impairment is that the trouble with seeing and perceiving the things effortlessly. In any case the treatment to visual impairment dependably relies upon the reason for the visual impairment. Roughly 80-90 percent of visual impairment can be counteracted by having a decent mix of instruction, great medicinal care and arrangement of glasses. Natural process reasons for visual debilitation measure preventable through right eating regime. Most instances of visual disability from eye measures preventable through early identification and appropriate treatment.

Many navigation technologies are developed throughout the years, however few are still living. A part of the explanation could also be because of a disconnection between engineering factors and a device's sensory activity and purposeful utility; that is, a device may work well in theory but would be too difficult or cumbersome in practice, to be adopted by the intended user. Few vital factors ought to be thought of once discussing the planning and implementation of technology for blind navigation.

\section{BACKGROUND WORK}

The various technologies has been developed for Blind Navigation.

\section{A. Navigation System Using RFID:}

Blind people need assistance in recognizing obstructions, discovering areas, and getting headings through making a course for achieve their goal. Upheld this industrious need, there is a route framework to encourage these necessities. They gave this route framework to blind students, workers, or visitors at King Saud University field space. The advances utilized as a part of our anticipated framework are: the visually impaired cell phone, RFID labels and Reader, GPS, content to discourse, voice Recognition, and Wi-Fi. The framework identifies the visually impaired area exploitation GPS, if net alliance is accessible and utilizes RFID labels attached outside and inside on the working inside the way, are arrange switches are utilized inside to find the area. The framework utilizes voice acknowledgment and content to discourse to speak with the heedless to guide him to his goal and to exhibit him the bearings. The outcomes demonstrate sensible execution in obstructions evasion and in blind guiding. 


\section{RFID Handheld Reader}

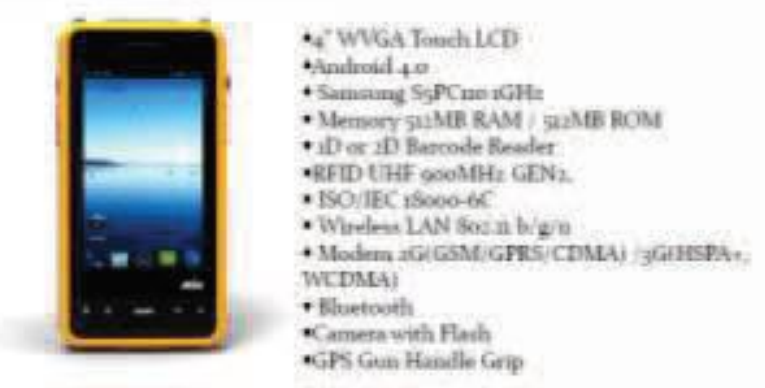

Figure 3: RFID Reader.

The framework made from the blind operated android mobile, the blind white stick with the RFID reader connected thereto, GPS, and RFID labels secured inside the points of interest for the blind way. The framework may utilize area network, WLAN, remote constancy, Wi-Fi, neighborhood (LAN) switches that are now dispersed all round the building. The framework fills in as takes after: the framework identifies the blinds area by GPS if potential or by reading the RFID label that is just about the RFID reader. The framework can speak with the visually impaired by voice letting him know concerning his area, at that point the client needs to raise the framework to move to such an extent of his goal (predefined like school, eatery, classroom, can, or office). The framework can guide him to move to the best possible bearing and separation to goal by electronic compass, GPS, and by perusing following recognized RFID labels in his way. Page 3

\section{B. Smart Assistive Navigation System for Blind:}

A Smart accommodating Navigation System for Blind and Visually Impaired individuals is composed and executed to have a safe and moderate route. Blind route frameworks are a few; however just a couple of the proposed frameworks are totally fruitful in tending to the necessities of visually impaired people to explore securely, well, and severally. Along these lines, a few happens to the dynamic works are specified and investigated. At long last, the look and in this manner the usage of the framework is appeared.

The fundamental goal of this task is to have a protected, ease route for the visually impaired/outwardly hindered individuals. To do that, to start with, visual interface between the Microsoft Kinect and furthermore the MATLAB is utilized; this interface helps in adjusting the inconspicuous obstructions by the client to voice noticed that may request and caution the previously mentioned client to maintain a strategic distance from these impediments. Second, this interface was improved with every one of its subtle elements into a self maintaining framework that would be upheld on the client, so it's deliberate even a little bit circumstances. Last however not least, an application that guarantees the correspondence between the client and his/her guide was made. This whole framework can go about as a virtual eye for the client. To actualize a route framework, we require a pack of sensors and furthermore a preparing unit to investigate every one of the information being caught by the given sensors. In this, the most appropriate sensor is the Microsoft kinect.

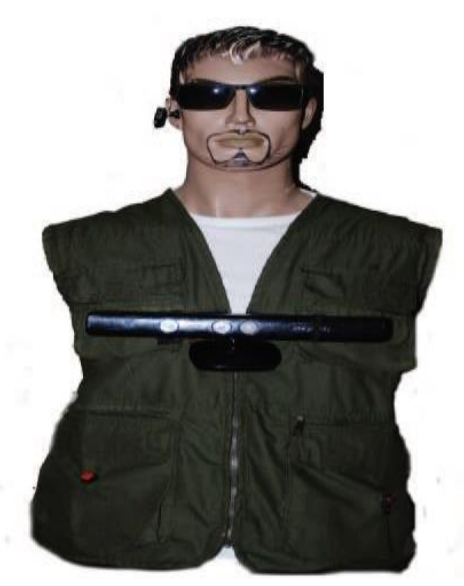

Figure 4: Microsoft kinect, final prototype.

Also, Arduino mega is required, which is a microcontroller that acknowledges serial interface. This interface is required in order to deal with the SOS catch which will initiate the versatile application and along these lines the switch which will flip the framework on and off. In addition, a transportable battery is required keeping in mind the end goal to create energy to the framework, correspondingly as a vest to convey all the over materials. Last however not minimum; Bluetooth headsets are utilized because of it's in an ideal situation to the client.

\section{Blind Navigation System Microsoft Kinect Camera:}

Maintaining a strategic distance from hindrances and exploring in like manner are real issues for outwardly weakened. They require help to pass securely. This paper presents relate deterrent maintaining a strategic distance from framework for blind individuals exploitation Kinect profundity camera. This accommodating innovation recognizes the medium before of the client exploitation Kinect profundity camera. The framework gets the profundity pictures from the Kinect camera and procedures it utilizing a 
windowing-based mean or normal system to recognize snags inside the filtered setting. Once the framework identifies a hindrance, it sends a voice input to the client through headphones. The testing is done with blind people. It demonstrates that this gadget may with progress manage them to sidestep obstructions securely. The current proposed framework comprises of three fundamental parts, for example, profundity camera, headphone and PC.

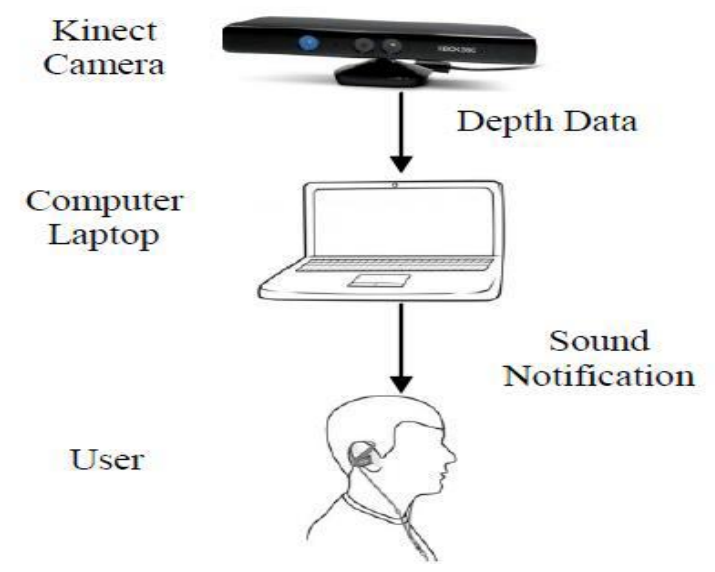

Figure 5: Flow of Data between Kinect, Laptop, and the User.

This shows a propelled consider on the innovation utilizing Kinetic camera which helps the visually impaired in distinguishing snags with an expansion of new technique for windowing-based mean strategy.

\section{Blind Navigation System on Haptic and Spatial Cognition:}

With the end goal of route for the visually impaired they proposed a framework which is a high accuracy route framework which ordinarily in view of haptic and the visually impaired spatial cognizance. This framework is joined with the spatial cognizance highlights of the visually impaired and the ultrawideband remote situating innovation to get the exact area with the assistance of various vibration modes like voice mode to direct the visually impaired individuals for the route. Page 4 The exactness of UWB innovation accomplished situating level is utilized to situating the outwardly debilitated for completing the course steerage. Distinctive vibration would tip the visually impaired in an exceptionally shrieking setting once voice provoke obstruction. This timetable will decrease the visually impaired intellectual weight and giving an extra different contrasting option to exact situating and courses inciting.

\section{E. Navigation techniques using $Q R$ codes:}

Blind route is an availability application that empowers oblivious in regards to utilize related humanoid. Cell phone is a clear way for indoor route with directions in sound kind. They require an anticipated model that is an inside route application for blinds that utilizations QR codes. It is produced for humanoid sensible telephones and won't require any additional equipment for route. It gives programmed directing help on pre-characterized courses for blind. QR codes square measure put on the ground segments once particular separation that goes about as partner contribution for current area location and route.

At whatever point a QR code is checked it furnishes the client with the data of the present area and requests that the client pick the goal so offers ideal and most brief way. Way discovering calculations help all through route at whatever point the deviation from the anticipated way is recognized it prompts the client and aides back to the right way by examination the present way with the produced way. The greater part of the headings all through the machine square measure gave in sound shape to the client. The interface of the apparatus is very much designed for blinds that make the advanced mobile phones simple and helpful for blind individuals. The client connects with the apparatus through a specific arrangement of simple signals for particular data sources and tasks. Toward the finish of this paper, they have performed examination between various condition of workmanship approaches and finished that our approach is a great deal of easy to use, financially savvy and made a considerable measure of right outcomes.

\section{F. ABAid: Navigation Aid for Blind:}

They presented ABAid, an extraordinary framework intended for blind or outwardly disabled people to explore, with prepared to deliver (COTS) cell phones, upheld top to bottom acoustic sensors and pivoting system strategies, this technique isn't exclusively the implies that of goliath comfort to hold, however conjointly fit for identifying snags before contacting them. Maybe a couple of their trials were intended to distinguish the space in the divider. With high exactness and stable action, ABAid is in a situation to help blind people move severally in genuinely uncomplicated circumstances. The guideline of this strategy is to use the ability of PDAs in discharging and accumulation the closer ultrasonic waves in the meantime. At the point when accumulation all the discharged waves, protests that square measure 
incorporating the client reflect the a piece of the waves that close by commotion square measure gathered by the electro-acoustic transducer and in this way the time slacks likewise are computed.

\section{G. BlinDar: An Invisible Eye for the Blind:}

A keen Electronic Traveling Aid (ETA) known as BlinDar has been arranged. This sensible managing ETA enhance the lifetime of visually impaired in light of the fact that it is all around outfitted with web of Things (IoT) and should help the outwardly impeded and oblivious to run while not imperative in close further as open conditions. BlinDar might be a to a great degree practical, dependable, snappy reacting, lightweight, low power overpowering and cost compelling gadget for the visually impaired. The ETAs have sensors that alarm the visually impaired preceding concerning the risk with sounds or vibrations. This paper proposes the gadget known as 'BlinDar' that is relate degree ETA.

It plans to enhance the lifetime of visually impaired and encourages them to self explore while not depending on some individual. This procedure gives choices like shoddy route stick which rapidly reacts when a deterrent is discovered, lightweight components coordinated on sticks, a wrist band is associated close to the keep on with a vibrator associated with that cautions him of the hindrance (for banging spots), RF Tx/Rx module is associated for finding the stick, a GPS module associated for area sharing, and MQ2 gas finder for recognition of flame inside the way. In places wherever the scopes of potholes are a ton in number than the specific snags, there's partner degree gigantic might want of sensible directing stick like BlinDar. The visually impaired needs a device that is a ton of sensible, utile, easy to understand and cost compelling like BlinDar might be a pleasant determination and most fitted different to white sticks and diverse ETA's.

\section{H. Blind Navigation using SONAR:}

This might be a piece of push to build up a route framework to help the outwardly disabled abuse estimating gadget. SONAR (Sound Navigation and Ranging) framework assumes a huge part in across the board applications in submerged situations. Here pressure is on the use of a proportional in period identification of impediment. We will probably construct a versatile, practical, lightweight framework for the oblivious in regards to facilitate their route while not help. At show, the current satellite route upheld GPS isn't sufficiently exact to Page 5 manage people on foot, especially around urban areas. The hindrance dismissing technique is outlining inside the framework, the execution of absolutely on the estimating gadget sensors utilized. The impact of their impediment on the arranged framework is given personally. The outcomes gave demonstrated that the execution of sonar gadgets once set appropriately fabricate the framework reasonable as a pilot for the outwardly impeded.

\section{COMPARATIVE STUDY}

Generally, when considering the navigation using RFID system there are few drawbacks using this system like these RFID are generally expensive and they try to read many tags at a time. Hence there might be a signal collision and they may result in the data loss. And it's not practically acceptable to install RFID readers at every possible location[1]. The current navigation system using depth kinetic camera has few limitations that they work at lower depth range, that is identifying obstacles at high range is not possible. Kinetic camera that is used now is the older version which does not reflect any light, and there is no face recognition in the system [2].

BlinDar is another system which made the lives of blind easier. This system basically uses IOT technology in building it. It's basically a smart stick build with many sensors and other objects. As known IOT based systems have many disadvantages which are still in resolving state and might not be that effectively used by the blind for the navigation purposes. Any drop or variation in internet would make the system not work as per designed [7].

There are many uses of QR codes like, identifying any locations or for making payments etc. QR codes are also used to find locations in indoor. It's practically not possible for a blind to scan the QR codes every often to find out the locations and move. Every time the person has to go out he has to pre define all the locations, and also having QR codes everywhere is also not possible. Hence this proposed system is not user friendly [5].

ABaid is a system designed for a mobile system which helps in detecting large obstacle devices; it consists of speakers, a microphone and a gyroscope. Usage of gyroscope has some disadvantages, say the gyro is expensive, and they become noisy if they are connected to sound. So, it's not cost effective so the user cannot afford to use it [6]. 
Blind navigation using sonar, the existing satellite navigation based on GPS is not that accurate enough to guide the blind people while walking. For this, primarily the sonar sensors are used. According to the test conducted SONAR sensors could only detect $40 \%$ of the obstacles which is a very less ratio and makes obvious to the users to accept that it cannot be used in the daily life of a visually impaired person [8].

A system based on haptic and spatial cognition for the visually impaired people. This system has real time positioning and precisions positioning, helping a visually impaired person to adjust in geographical environment with the help of sounds and diversity vibrations of prompts helping the blind people. This is an advanced and an user friendly technology that can be used for navigation [4].

At last going to a proficient Assistive Technology (AT) to help blind individuals to keep away from obstruction utilizing Kinect profundity camera, and afterward another approach that is windowing-based mean technique is utilized. This framework can supplant the various innovations, which is a costly decision by correlation and thinking about that white stick is lighter, less expensive and no need of any power. Favorable position of this is to begin with, this framework can manage the outwardly debilitated individuals where to go. Second the scope of this framework is significantly more i.e., 4 meter long, where scope of the stick is 1-1.5 meter. Moreover, the framework has the help to start programs like read works, track or perceive individuals. At last, this framework can be enhanced by including a haptic communication notwithstanding the sound feedback [3].

\section{CONCLUSION}

The Internet of Things (IoT) is a very promising technique. It is expounded as the network of real or virtual entities embedded with electronics, sensors and software's with network connectivity which enables these entities to collect and exchange data, thus providing connectivity at anytime. In a country like India, where the numbers of potholes are more in number than the actual obstacles, there is an immense need of smart guiding for visually impaired. The main drawback of the blind people is that they deprive themselves of what they deserve. The blind needs a device which is smarter, multipurpose, user friendly and cost effective. The scientists and researchers are working upon various technologies to eliminate the environmental, social and navigational problems faced by the blind and visually impaired people. Some of the tools are inaccessible by most of the visually impaired people because of cost and it's usage. The combination of various helpful solutions in a single system could be a great challenge. The integration of various assistive solutions in an individual system is a great challenge. Future improvements will consist of making the system able to work at better range, and with robustness to strong-light conditions of the system. Page 6

\section{References}

[1] Rachid Sammouda, Ahmad AlRjoub, "Mobile Blind Navigation System Using RFID”, Computer \&amp; Information Technology (GSCIT), 2015 Global Summit.

[2] Michel Owayjan, Ali Hayek, Hassan Nassrallah, Mohammad Eldor, "Smart Assistive Navigation System for Blind and Visually Impaired Individuals", 2015 International Conference on Advances in Biomedical Engineering (ICABME).

[3] Ali Ali, Mohammad Abou Ali, "Blind Navigation System for Visually Impaired Using WindowingBased Mean on Microsoft Kinect Camera", 2017 Fourth International Conference on Advances in Biomedical Engineering (ICABME).

[4] Jiangang Ma, Jianghua Zheng, "High Precision Blind Navigation System Based on Haptic and Spatial Cognition", 2017 2nd International Conference on Image, Vision and Computing.

[5] Affan Idrees, Zahid Iqbal, Maria Ishfaq, "An efficient indoor navigation technique to find optimal route for blinds using QR Codes", 2015 IEEE 10th Conference on Industrial Electronics and Applications (ICIEA).

[6] Zehui Zheng, Weifeng Liu, Rukhsana Ruby, Yongpan Zou, Kaishun Wu, "ABAid: Navigation Aid for Blind People Using Acoustic Signal”, 2017 IEEE 14th International Conference on Mobile Ad Hoc and Sensor Systems.

[7] Zeeshan Saquib, Vishakha Murari, Suhas N Bhargav, "BlinDar: An Invisible Eye for the Blind People", 2017 2nd IEEE International Conference On Recent Trends In Electronics Information \&amp; Communication Technology.

[8] Kavitha Viswanathan, Prof. Sharmila Sengupta, "Blind Navigation Proposal using SONAR", 2015 IEEE Bombay Section Symposium (IBSS). 